\title{
Evaporation and condensation of multicomponent electronic cigarette droplets and conventional cigarette smoke particles in an idealized G3-G6 triple bifurcating unit
}

\author{
Y. Feng ${ }^{a}$, C. Kleinstreuer ${ }^{a, b, *}, A$. Rostami $^{c}$ \\ a Department of Mechanical and Aerospace Engineering, North Carolina State University, Raleigh, NC 27695, USA \\ b Joint UNC-NCSU Department of Biomedical Engineering, North Carolina State University, Raleigh, NC 27695, USA \\ c Altria Client Services Inc., Research, Development and Engineering, 601 East Jackson Street, Richmond, VA 23219, USA
}

\section{A R T I C L E I N F O}

\section{Article history:}

Received 19 June 2014

Received in revised form

1 November 2014

Accepted 6 November 2014

Available online 18 November 2014

Keywords:

Electronic cigarette (EC)

Conventional cigarette smoke particles

(CSPs)

Multicomponent droplets

Deposition

Droplet-vapor interaction modeling

Multicomponent Mixture plus Discrete

Droplet (MM-DD) model

\begin{abstract}
A B S T R A C T
The present research study is intended to provide fundamental understanding of the dynamics and transport of aerosols from an e-cigarette in an idealized tubular G3-G6 respiratory tract model. A computational model has been developed that includes the effects of hygroscopic growth as well as evaporation from multicomponent aerosol droplets. The aerosols investigated usually contain carrier solvents such as propylene glycol (PG) and glycerol, along with water, nicotine, and flavors. An experimentally validated computational fluid-particle dynamics (CF-PD) model is presented, which for the first time is capable of simultaneously simulating interactive, multicomponent droplet-vapor dynamics with evaporation and/or condensation. As a first step to accomplish such complex numerical simulations, an idealized G3-G6 triple bifurcating unit (TBU) has been selected. The results are compared with the conventional smoke particles (CSPs) as well as solid particles. Parametric analysis and comparisons of the evaporation/condensation dynamics for EC-droplets vs. cigarette smoke particles were performed, including the effects of different droplet initial diameter, composition, temperature, and ambient relative humidity. The results indicate that EC-droplets, being more hygroscopic than cigarette smoke particles, tend to grow larger in maximum size in a typically highly humid environment. Additionally, a correlation for the growth ratio of EC-droplets in TBUs is proposed.
\end{abstract}

(c) 2014 Elsevier Ltd. All rights reserved.

\section{Introduction}

In general, realistic and accurate computer simulation models are very useful and cost-effective tools for predicting the transport and deposition of aerosols. Such models improve the fundamental scientific understanding of the dynamics and transport of aerosols. Quantitative results generated from such models could be invaluable to regulatory agencies, healthcare providers and manufacturers. It can also better inform and strengthen the science base needed to evaluate and regulate electronic cigarettes (Chen \& Husten, 2014; Chen, 2013).

\footnotetext{
* Corresponding author at: Department of Mechanical and Aerospace Engineering, North Carolina State University, Raleigh, NC 27695, USA. Tel.: +1 919515 5216; fax: +1 9195157968 .

E-mail address: ck@ncsu.edu (C. Kleinstreuer).
} 


\begin{tabular}{|c|c|c|c|}
\hline \multicolumn{2}{|c|}{ Nomenclature } & $u_{d}$ & droplet velocity \\
\hline$c_{p}$ & liquid specific heat & Y & mass fraction \\
\hline$C_{M}, C_{T}$ & Knudsen number correction factors & \multirow{3}{*}{\multicolumn{2}{|c|}{ Greek symbols }} \\
\hline$D_{1}$ & inlet diameter of G3-G6 TBU & & \\
\hline$D_{a-v, s}$ & diffusivity & & \\
\hline$d_{d}$ & droplet diameter & \multirow{2}{*}{$\begin{array}{l}\alpha_{m} \\
\alpha_{T}\end{array}$} & \multirow{4}{*}{$\begin{array}{l}\text { mass accommodation coefficient } \alpha_{m}=1 \\
\text { thermal accommodation coefficient } \alpha_{T}=1 \\
\text { activity coefficient of species } s \\
\text { mean free path }\end{array}$} \\
\hline$d_{d, i n i}$ & initial droplet diameter & & \\
\hline$d_{d, \max }$ & maximum droplet hygroscopic diameter & $\gamma_{s}$ & \\
\hline$D E$ & deposition efficiency & $\lambda$ & \\
\hline EC & electronic cigarette & $\nu$ & kinetic molecular viscosity \\
\hline$H$ & enthalpy & $\rho_{a-v}$ & air-vapor mixture density \\
\hline$h_{G 3}$ & $\begin{array}{l}\text { initial distance between droplet location and } \\
\text { G3 inlet centroid }\end{array}$ & & $\begin{array}{l}\text { droplet density } \\
\text { viscous shear stress }\end{array}$ \\
\hline$j_{S}$ & $\begin{array}{l}\text { evaporation/condensation mass flux of the sth } \\
\text { component }\end{array}$ & $\begin{array}{l}\sigma \\
\Phi\end{array}$ & $\begin{array}{l}\text { surface tension } \\
\text { dissipation function }\end{array}$ \\
\hline $\begin{array}{l}k_{a-v} \\
k_{d}\end{array}$ & $\begin{array}{l}\text { air-vapor mixture thermal conductivity } \\
\text { droplet thermal conductivity }\end{array}$ & & dynamic viscosity \\
\hline$K_{s}$ & $\begin{array}{l}\text { correction factor for Kelvin effect } K_{s}=\exp \left[4 \sigma_{s}\right. \\
\left.M_{s} /\left(\Re \rho_{s} d_{d} T_{d}\right)\right]\end{array}$ & \multicolumn{2}{|c|}{ Subscripts } \\
\hline Kn & Knudsen number $K n=2 \lambda_{a-v} / d_{d}$ & $a b s$ & absolute \\
\hline$L$ & latent heat of evaporation/condensation & air & dry air \\
\hline$m_{d}$ & droplet mass & $a-m$ & air-mucus layer \\
\hline$M_{S}$ & molecular weight of species $s$ & $a-v$ & air-vapor mixture (continuous phase) \\
\hline$N_{d, \text { cell }}$ & $\begin{array}{l}\text { the total droplet number in a specific } \\
\text { mesh cell }\end{array}$ & $\begin{array}{l}\text { cond } \\
d\end{array}$ & $\begin{array}{l}\text { condensation } \\
\text { droplet or liquid phase }\end{array}$ \\
\hline$N_{d e p}$ & number of deposited droplets & env & environmental or ambient \\
\hline $\mathrm{Nu}$ & $\begin{array}{l}\text { Nusselt number } \quad N u=\left(1+R e_{d} P r\right)^{1 / 3} \max \\
{\left[1, R e_{d}^{0.077}\right]}\end{array}$ & eq & $\begin{array}{l}\text { equilibrium } \\
\text { water }\end{array}$ \\
\hline $\operatorname{Pr}$ & Prandtl number $\operatorname{Pr}=c_{p} \mu / k_{a-v}$ & & tensor index \\
\hline$P_{v s, s a t}$ & saturated vapor pressure & & tensor index \\
\hline $\operatorname{Re}_{d}$ & $\begin{array}{l}\text { droplet Reynolds number } \operatorname{Re}_{d}=\rho_{a-v} \mid \vec{v}_{a-v}- \\
\vec{v}_{d} \mid d_{d} / \mu_{a-v}\end{array}$ & & mass \\
\hline $\mathfrak{R}_{s}$ & species gas constant & & thermal \\
\hline$\Re$ & universal gas constant & tot & total \\
\hline RH & relative humidity & $v$ & vapor phase \\
\hline Sc & Schmidt number $S c=v / D_{a-v}$ & & vapor-droplet \\
\hline$S h$ & $\begin{array}{l}\text { Sherwood number } \quad S h=\left(1+R e_{d} S c\right)^{1 / 3} \\
\max \left[1, R e_{d}^{0.077}\right]\end{array}$ & vsat & saturated vapor \\
\hline$t$ & time & w & wall \\
\hline$T$ & temperature & & \\
\hline$U$ & continuous phase (air-vapor mixture) velocity & & \\
\hline
\end{tabular}

An electronic cigarette (EC) typically consists of a flow sensor, a solution (e-liquid) storage unit and a battery-powered heater, which vaporizes the liquid (Brown \& Cheng, 2014). The e-liquid mixture usually contains carrier solvents such as propylene glycol (PG) and glycerol, along with water, nicotine and flavors (Cheng, 2014). Aerosols formed from ECs are considerably different from conventional cigarettes, in terms of chemical composition (Polosa et al., 2013; Cheng, 2014). These differences could result in different hygroscopic growth and evaporation rates when exposed to highly humid environments or when diluted substantially with air. As a result, the dynamic behavior and deposition rate of EC aerosol droplets may also be considerably different from cigarette smoke particles.

The effects of evaporation/condensation of the volatile components in conventional cigarette smoke aerosols in the human respiratory system have been investigated numerically using computational fluid-particle dynamics (CF-PD) simulations. For example, Longest and Xi (2008) studied evaporation and condensation behavior of conventional cigarette smoke particles (CSPs) in still air and their depositions in a respiratory system. They concluded that ambient $R H$ variations, e.g., air super-saturation, can contribute to enhanced CSP deposition. However, they did not discuss the droplet temperature variations during the evaporation and condensation process. Zhang et al. (2012a) compared the depositions of CSPs and Next-Generation Product (NGP) droplets in a human lung-airway model, and discovered that the composition change can significantly influence the hygroscopic growth process of droplets. They also found that at $R H=99.5 \%$, CSPs grow and reach the equilibrium state within negligible time durations. 
However, they did not consider the influence of evaporation or condensation at the droplet surface on the local vapor-species concentrations. Additionally, they consider water as the only evaporative components in multicomponent droplets.

Concerning the use of triple bifurcating units (TBUs), Zhang et al. (2006a) modeled the ambient $R H$ influence on the hygroscopicity of micron droplets and their deposition in human upper lung airways. They claimed that vaporizing droplets are more confined when compared with constant-diameter particles. Zhang et al. (2006b) also simulated the evaporative and hygroscopic effects of micron saline droplets on deposition from mouth to a Weibel G0-G3 TBU under steady transitional inspiratory flow conditions. They discovered that parameters such as $R H$, droplet composition and environmental temperature greatly influence droplet shrinkage or growth, and in turn affect deposition locations in human upper airways. However, they did not provide detailed parametric analyses for the G0-G3 TBU region.

As mentioned, in order to gain some basic physical insight and set the stage for more comprehensive numerical simulations, an idealized triple bifurcating unit (TBU), being representative of a human bronchial lung airway segment (Feng \& Kleinstreuer, 2014), has been selected. EC-aerosols consist of vapors and droplets. Based on an updated literature review, no research has been done on modeling EC droplet-vapor aerosols of different types in TBUs, especially with an emphasis on the multicomponent droplet-vapor interaction dynamics. Thus, the research objectives are:

(1) to develop and validate a novel CF-PD model for multicomponent droplet hygroscopic growth in a representative TBU;

(2) to find out dominant parameters (or properties) which influence the dynamics of EC-droplet evaporation/condensation in the G3-G6 TBU; and

(3) to understand the effects of evaporation/condensation characteristics of EC-droplets and CSPs on the transport dynamics and deposition patterns in the G3-G6 TBU.

In this study, a Multicomponent Mixture plus Discrete Droplet (MM-DD) model is introduced for the simulation of droplet-vapor mixture transport and deposition in the G3-G6 TBU, considering droplet evaporation/condensation effects as well as droplet-vapor coupling. Specifically, parametric analysis for EC-droplet size-change was carried out for droplets with different initial diameters, initial compositions, initial droplet temperature, and ambient $R H$-values. Furthermore, hygroscopic effects of EC-droplets and CSPs on their transport and deposition patterns for steady flow were examined and compared with those of solid particles. A correlation based on the numerical results for droplet hygroscopic growth is provided, which facilitates comparisons with future experimental measurements.

\section{Theory}

EC vapor-droplet interaction is a challenging, multidisciplinary issue. It involves heat and mass transfer, fluid dynamics, and chemical reaction kinetics (Sirignano, 2010). In an extension of the previous work by Zhang et al. (2012a, 2012b), we incorporate the actual droplet-vapor mechanisms, subject to justifiable assumptions and simplifications in light of realism, accuracy and computing cost. Thus, we propose a Multicomponent Mixture plus Discrete Droplet (MM-DD) model, which for the first time is capable of simultaneously simulating multicomponent droplet-vapor and airflow dynamics in respiratory systems. Figure 1 depicts the flowchart of the numerical model.

\subsection{Assumptions}

In order to arrive at a realistic and accurate computer simulation model which is also manageable in terms of required resources, the following assumptions and simplifications were made for the MM-DD model development:

(1) One-way coupling between air and vapors. As vapor species form dilute suspensions in the air, interactions between air and vapor species are assumed to be one-way coupled, indicating that vapor influences on the air are neglected.

(2) Air-vapor mixture properties. Because the mass fractions of saturated vapor species are relatively small, the viscosity $\nu_{a-v}$ and mean free path $\lambda_{a-v}$ of the air-vapor mixture can be approximated by the properties of pure air, i.e., $\nu_{a i r}$ and $\lambda_{\text {air }}$, respectively. Furthermore, the variations of local mass fractions of vapor species have no influence on the air-vapor mixture properties, i.e., mainly density $\rho_{a-v}$.

(3) Well-mixed nano-/micro-droplets. Based on rapid mixing instantaneous heat conduction and mass diffusion within the droplets (Zhang et al., 2006a; Longest \& Xi, 2008), the droplet temperature and mole fractions of liquid ingredients are spatially constant but vary with time.

(4) Neglect droplet-droplet interactions. The effects of coagulation, cloud formation and droplet charge are insignificant because the EC aerosol suspension is dilute.

\subsection{Governing equations for continuous phase (air-vapor mixture) transport}

Describing the air-vapor mixture of EC-aerosols as a single continuous phase, we employed the conservation of mass, momentum, and energy to solve the air-vapor mixture transport phenomena, as well as the advection-diffusion equations 


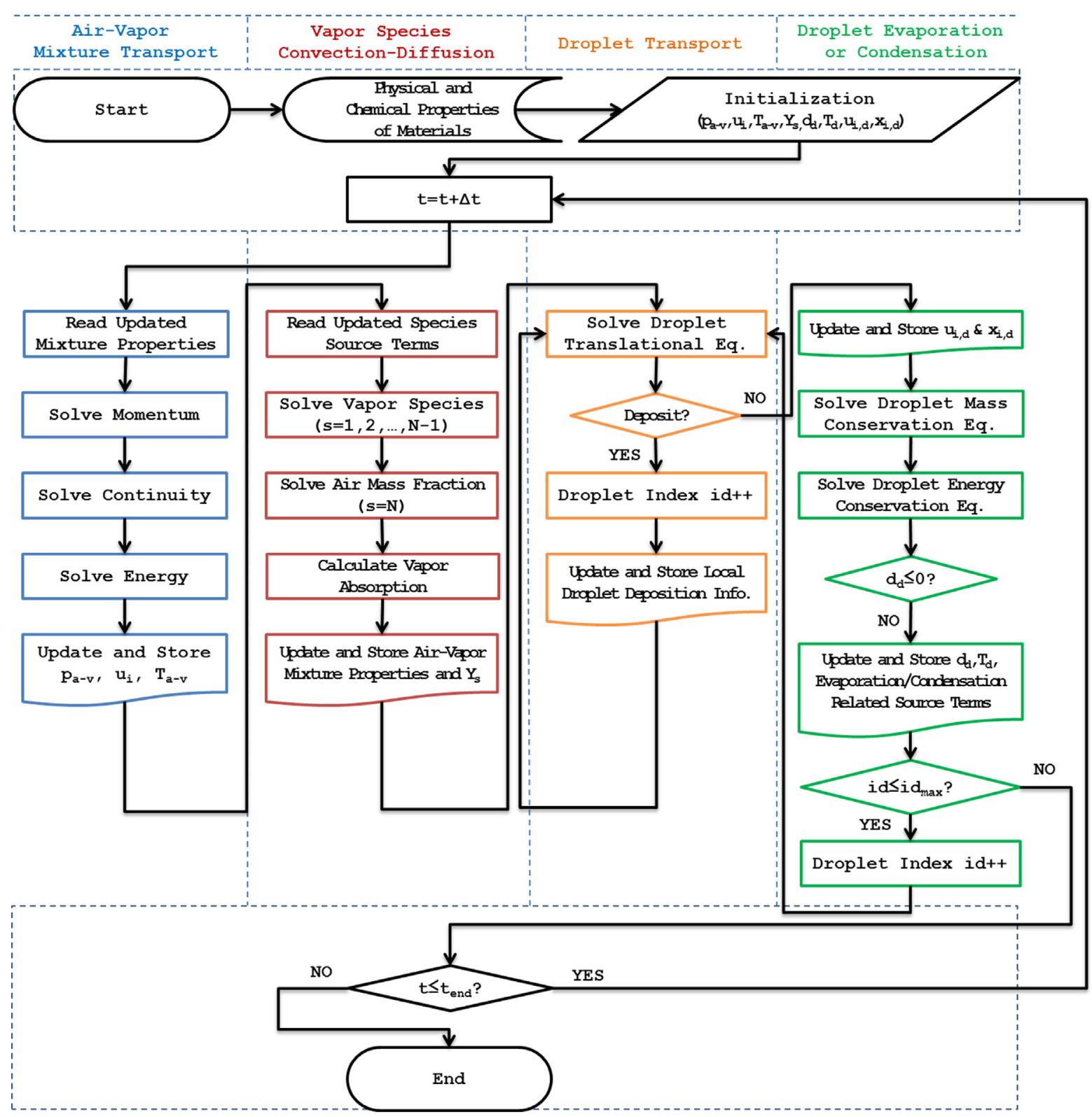

Fig. 1. Flowchart of the MM-DD model with simultaneous droplet-vapor interactions.

for vapor species transport. Specifically, according to the inlet conditions, laminar flow regimes are expected in the G3-G6 TBU (i.e., $R e_{\text {in }} \leq 61$ at the $\mathrm{G} 3$ inlet, corresponding to the mouth air flow rate of $37.5 \mathrm{ml} / \mathrm{s}$ when smoking).

Droplet-vapor interaction will be realized via:

- introducing source terms into the energy equation and vapor species transport equations (see Eqs. (1)-(4)); and

- employing the local vapor mass fraction in droplet mass conservation equation (see Eq. (6)).

Specifically, the energy equation of air-vapor mixture can be written as

$$
\frac{\partial\left(\rho c_{p} T\right)_{a-v}}{\partial t}+\frac{\partial\left(\rho c_{p} u_{j} T\right)_{a-v}}{\partial x_{j}}=\frac{\partial}{\partial x_{j}}\left[\left(k_{a-v}+\frac{\rho_{a-v} c_{a-v, p} \nu_{T}}{P r_{T}}\right) \frac{\partial T_{a-v}}{\partial x_{j}}\right]+\Phi+\frac{\partial}{\partial x_{j}}\left[\sum_{s=1}^{N} h_{s} \rho_{a-v}\left(\tilde{D}_{a-v}+\frac{\nu_{T}}{S c_{T}}\right) \frac{\partial Y_{s, v}}{\partial x_{j}}\right]+S_{v-d}^{(E)}
$$


In Eq. (1), the energy source term $S_{v-d}^{(E)}$ is due to the latent heat of evaporation or condensation which are released or absorbed by the droplets per local mesh cell:

$$
S_{v-d}^{(E)}=\sum_{s=1}^{N} S_{v-d, s}^{(E)}=\left\{\sum_{i=1}^{N_{d, c e l l}}\left[\left(\sum_{s=1}^{N} L_{s} \overline{j_{s}}\right) A_{d}\right]_{i}\right\} / V_{\text {cell }}
$$

where $N_{d, c e l l}$ is the total droplet number in a specified mesh cell.

The governing equation for the advection and diffusion of the sth EC-vapor species is

$$
\frac{\partial\left(\rho_{a-v} Y_{s, v}\right)}{\partial t}+\frac{\partial}{\partial x_{j}}\left(\rho_{a-v} u_{j} Y_{s, v}\right)=\frac{\partial}{\partial x_{j}}\left[\rho_{a-v}\left(\tilde{D}_{a-v, s}+\frac{\nu_{T}}{S c_{T}}\right) \frac{\partial Y_{s, v}}{\partial x_{j}}\right]+S_{v-d, s}^{(Y)}
$$

Here, $S c_{T}=0.9$ is the turbulent Schmidt number for $Y_{S, v}$ (Zhang et al., 2012b; Berthier \& Silberzan, 2006), $\nu_{T}$ is the turbulence viscosity, and $\tilde{D}_{a-v, s}$ is the molecular diffusivity of the sth vapor species in the air-vapor mixture. The local vaporized/ condensed vapor mass flow rate of the EC-aerosol components are added to its advection-diffusion equation as an averaged source term $S_{v-d, s}^{(Y)}\left(\mathrm{kg} \mathrm{m}^{-3} \mathrm{~s}^{-1}\right)$. It will be updated in each flow time step and can be given by

$$
S_{v-d, s}^{(Y)}=\frac{\int_{t_{i, s t a r s}}^{t_{i, \text { stars }}+\Delta t_{f}}\left(\sum_{i=1}^{N_{d, \text { cell }}}\left(\overline{j_{s}} A_{d}\right)_{i}\right) d t_{d}}{V_{\text {cell }} \Delta t_{f}}
$$

where $\overline{j_{s}}$ is the average evaporation/condensation mass flux normal to the droplet surface of sth component (i.e., $\overline{j_{S}}>0$ for evaporation and $\overline{j_{s}}<0$ for condensation); $A_{d}$ is the droplet surface area.

\subsection{Governing equations for multicomponent droplets}

The Lagrangian scheme is chosen to track multicomponent droplets, neglecting rotational motion. The governing equations for discrete droplets are the translational equation of droplets as well as the mass and energy conservation laws for droplets (Longest \& Xi, 2008; Zhang et al., 2012a, 2012b).

\subsubsection{Translational equation of droplets}

$$
\frac{d}{d t}\left(m_{d} u_{d, i}\right)=F_{i}^{D}+F_{i}^{L}+F_{i}^{B M}
$$

where $F_{i}^{D}, F_{i}^{L}$, and $F_{i}^{B M}$ are the Stokes drag force, lift force, and Brownian motion induced force on the droplet, respectively (Longest \& Xi, 2008; Zhang et al., 2012a, 2012b).

\subsubsection{Mass conservation of droplets}

$$
\frac{d m_{d}}{d t}=-\sum_{s=1}^{N} \int_{\text {surf }} j_{s} d A \approx-\sum_{s=1}^{N}\left(\overline{j_{s}} A_{d}\right)
$$

where the average evaporation/condensation mass flux $\overline{j_{s}}$ is given by Turns (1996) as

$$
\overline{j_{s}}=\rho_{a-v} C_{m} \frac{S h \tilde{D}_{a-v, s}}{d_{d}} \ln \frac{1-Y_{s, v, \text { cell }}}{1-Y_{s, v s u r f}}
$$

in which $S h$ is the Sherwood number (see the nomenclature for definition). $Y_{s, v s u r f}$ and $Y_{s, v, c e l l}$ are the mass fractions of the sth vapor phase at the droplet surface and at the center of the cell where the droplet currently resides. The correction factor $C_{m}$ for submicron droplets, considering the non-continuum effects, can be expressed as (Zhang et al., 2006b)

$$
C_{m}=\frac{1+K n}{1+\left(\left(4 / 3 \alpha_{m}\right)+0.377\right) K n+\left(4 / 3 \alpha_{m}\right) K n^{2}}
$$

Here, $K n$ is the Knudsen number, defined as $K n=2 \lambda_{a-v} / d_{d}$ and $\lambda_{a-v}$ is the mean free path of the air-vapor mixture surrounding the droplet; $\alpha_{m}$ is the mass accommodation coefficient. A value of $\alpha_{m}=1$ was used in the present study (Hinds, 1999). The derivation of Eq. (7) is based on the assumption that the distance between the droplet mass center and the mesh cell center is much larger than the droplet radius. Specifically, $Y_{s, v, \text { cell }}$ is determined by the advection-diffusion equation (see Eq. (3)), to realize the droplet-vapor interaction modeling. Furthermore, $Y_{s, v s u r f}$ can be calculated by

$$
Y_{s, v \text { surf }}=\frac{\rho_{s, v \text { surf }}}{\rho_{a-v}}=\frac{\left(P_{s, v s u r f} / \mathfrak{R}_{s} T_{d}\right)}{\rho_{a-v}}=\frac{\gamma_{s} K_{s} X_{s, d} P_{s, v s a t}\left(T_{d}\right)}{\rho_{a-v}\left(\mathfrak{R} / M_{s}\right) T_{d}}
$$

where $\rho_{s, v s u r f}$ is the equivalent density of vapor species $s$ in the air-vapor mixture at the droplet surface, and $\gamma_{s}$ is the activity coefficient of species $s$, which is a correction of the evaporation/condensation characteristics of a certain liquid component due to the molecular bonding changes between in the pure droplets and in the multicomponent droplets (Tu \& Ray, 2005); 
$X_{s, d}$ is the mole fraction of the sth component in the droplet, $\mathfrak{R}_{s}$ is the species gas constant, $T_{d}$ is the droplet temperature, and $P_{s, v s a t}$ is the temperature-dependent saturation pressure of the pure sth species. Here, $\gamma_{s}$ and $X_{s, d}$ serve as corrections to the vapor pressure at the surface of multicomponent droplets (Raoult's law). In addition, $K_{s}$ is the Kelvin effect correction factor (Hinds, 1999), which is given as (Longest \& Xi, 2008; Zhang et al., 2012a, 2012b)

$$
K_{s}=\exp \left[4 \sigma_{s} M_{s} /\left(\Re \rho_{s} d_{d} T_{d}\right)\right]
$$

where $\sigma_{s}$ is the surface tension of component $s$ at the droplet surface.

\subsubsection{Energy conservation of droplets}

$$
m_{d} c_{p, d}\left(d T_{d} / d t\right)=C_{T}\left(\frac{k_{a-v} N u}{d_{d}}\right)\left(T_{a-v, c e l l}-T_{d}\right) A_{d}-\left(\sum_{s=1}^{N} L_{s} \overline{j_{s}}\right) A_{d}
$$

where $N u$ is the Nusselt number (see the Nomenclature for definition) and $L_{s}$ is the latent heat of liquid-vapor phase transition of the sth species ( $L_{s}$ is always larger than zero). Also, $C_{T}$ is the correction factor for submicron droplets:

$$
C_{T}=\frac{1+K n}{1+\left(\left(4 / 3 \alpha_{T}\right)+0.377\right) K n+\left(4 / 3 \alpha_{T}\right) K n^{2}}
$$

where $\alpha_{T}$ is the thermal accommodation coefficient. The value of $\alpha_{T}=1$ was used in the present study (Hinds, 1999).

\section{Numerical simulation}

\subsection{Geometry and mesh}

A representative triple bifurcation bronchial airway model was selected for this study (see Fig. 2), where the dimensions are representative for adults with a lung volume of $3500 \mathrm{~mL}$. To represent bifurcating airways starting from different generations, the triple bifurcating airway geometry was scaled to duplicate the hydraulic diameter $D_{1}$ of the first bifurcation of different generations. For example, $D_{1}=0.6 \mathrm{~cm}$ represents the G3-G6 TBU.

For the numerical simulation a structured, multi-block, body-fitted hexahedral mesh was developed (see Fig. 2). Mesh independence tests have been successfully executed and presented in published papers (e.g., Zhang \& Kleinstreuer, 2001). The final mesh contained 628,712 cells, 655,822 nodes, and 1,912,466 faces.

\subsection{Boundary conditions}

For steady-state simulations, the averaged inlet velocity was determined from the air flow at the mouth, i.e., $Q_{i n}=37.5 \mathrm{ml} / \mathrm{s}$, which is a representative value of the mean flow rate while smoking (Zhang et al., 2012a). To facilitate the determination of inlet conditions, three assumptions were introduced: (a) the lung morphology is identical to the idealized model proposed by Weibel (1963); (b) the mass flow rate in each branch at the same generation is evenly
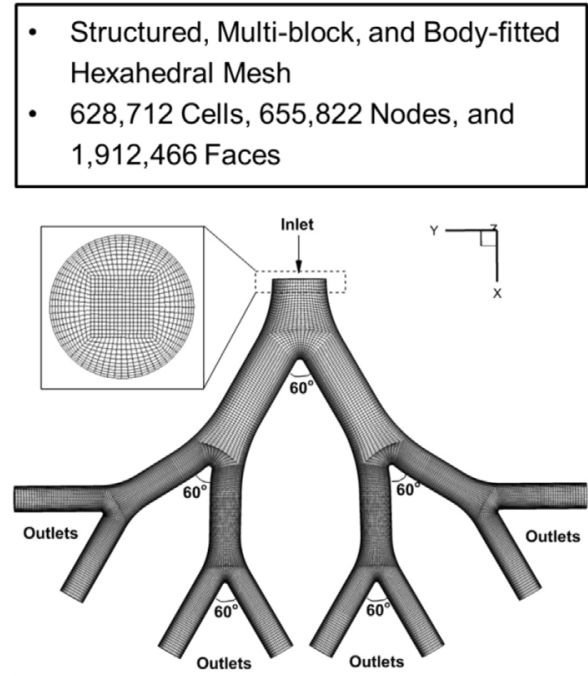

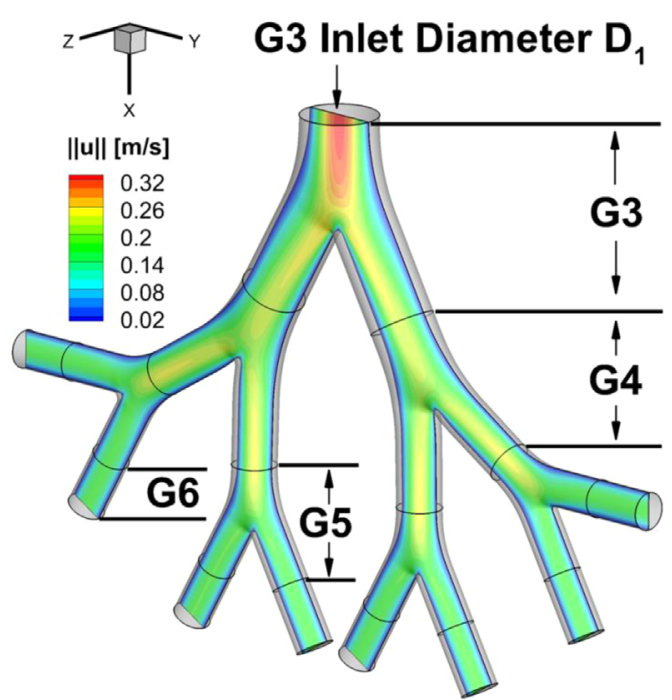

Fig. 2. Geometry, mesh, and airflow field of Weibel's G3-G6 triple bifurcating unit (TBU). 
distributed; and (c) the inlet flow is fully-developed with a parabolic velocity profile. Therefore, the inlet Reynolds number at G3 is 61 and the corresponding airflow field is shown in Fig. 2 as well.

Random-parabolic droplet/particle distributions with a total number of 11,328 were generated at the G3 inlet, using an in-house MatLab code (Feng \& Kleinstreuer, 2014). Additionally, a constant wall temperature of $37{ }^{\circ} \mathrm{C}$ (i.e., $310.15 \mathrm{~K}$ ) was assumed at the walls and uniform (zero) pressure was applied at the G6 outlets. Droplet deposition occurs when its mass center comes within the droplet radius from the wall (Zhang et al., 2012a, 2012b; Feng \& Kleinstreuer, 2014).

\subsection{Numerical setup}

The computational results of the governing equations with appropriate boundary conditions were achieved by using a user-enhanced, commercial finite-volume based program, i.e., ANSYS Fluent 14.5 (Ansys Inc., Canonsburg, PA). The numerical simulations were performed on a local Dell Precision T3500 workstation with 12 GB RAM and four 3.33 GHz CPUs as well as on a local Dell Precision T7500 workstation with 40 GB RAM and 123.33 GHZ CPUs. The newly developed user-enhanced UDFs are able to perform the following tasks:

(1) Recovering Brownian motion induced fluctuation velocities of nano-scale droplets.

(2) Solving droplet mass and energy conservation equations (i.e., evaporation/condensation).

(3) Recording local deposition information of vapors and droplets.

\section{Model validation}

To validate our multicomponent droplet evaporation/condensation model, we simulated the hygroscopic growth of conventional cigarette smoke particles (CSPs) by treating conventional smoke droplets as a water solution with soluble components. Specifically, it was assumed that the mainstream CSPs contain mass fractions of $41.7 \%$ for water and $58.3 \%$ for soluble components with molecular weight $340 \mathrm{~g} / \mathrm{mol}$ (Ishizu et al., 1980; Longest \& Xi, 2008). The model validation results are given in Fig. 3, where the present simulations for the CSPs hygroscopic characteristics agree well with previous simulation results (Zhang et al., 2012a) and experimental data (Li \& Hopke, 1993).

It should be noted that the droplet-growth ratio is very sensitive to even small variations in the physical and chemical properties of water. Hence, errors introduced via property correlations (e.g., saturated vapor pressure, density), which can lead to minor changes in the saturated vapor pressures or partial pressures of volatile species, will have a noticeable impact on the droplet equilibrium status, thereby influencing the droplet-size changes.

\section{Results and discussion}

\subsection{Dominant parameters impacting hygroscopic behavior of EC-droplets and liquid CSPS}

In this section, we compared the hygroscopic characteristics between two types of conventional (liquid) cigarette smoke particles (CSPs \#1 and CSPs \#2) and EC-droplets $\left(50 \mathrm{~nm} \leq d_{d, i n i} \leq 3200 \mathrm{~nm}\right.$ ) with different compositions (see Table 1 ).

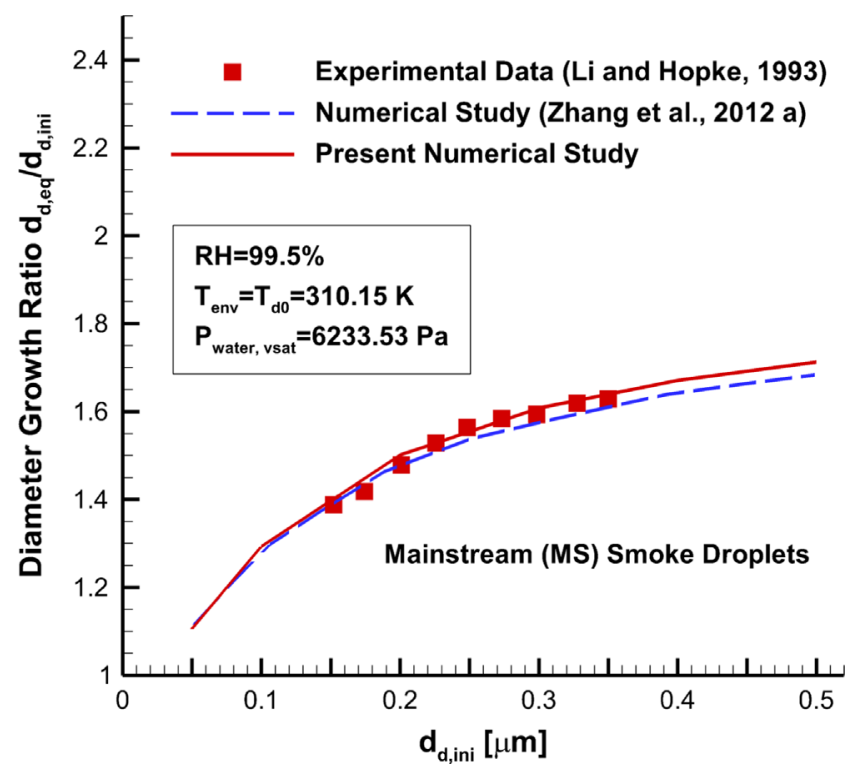

Fig. 3. MM-DD model validation: droplet maximum growth ratio vs. initial droplet size due to hygroscopic growth for conventional mainstream smoke droplets. 
Table 1

Composition of different EC-droplets (mass fraction), used for modeling.

\begin{tabular}{llllll}
\hline & \multicolumn{2}{l}{ Mass fraction of individual component } & & $\begin{array}{c}\text { Approximate nicotine } \\
\text { concentration in } \\
\text { e-liquid [mg/ml] }\end{array}$ \\
\cline { 2 - 5 } & Water & Glycerol & PG & Nicotine & 5 \\
EC-Droplet \#A1 & 0.15 & 0.498 & 0.347 & 0.005 & 10 \\
EC-Droplet \#A2 & 0.15 & 0.498 & 0.342 & 0.01 & 20 \\
EC-Droplet \#A3 & 0.15 & 0.498 & 0.332 & 0.02 & 30 \\
EC-Droplet \#A4 & 0.15 & 0.498 & 0.322 & 0.03 & 40 \\
EC-Droplet \#A5 & 0.15 & 0.498 & 0.312 & 0.04 & 20 \\
EC-Droplet \#B1 & 0.25 & 0.398 & 0.332 & 0.02 & 20 \\
EC-Droplet \#B2 & 0.15 & 0.498 & 0.332 & 0.02 & 20 \\
EC-Droplet \#B3 & 0.05 & 0.598 & 0.332 & 0.02 & 20 \\
EC-Droplet \#C1 & 0.15 & 0.598 & 0.232 & 0.02 & 20 \\
EC-Droplet \#C2 & 0.15 & 0.498 & 0.332 & 0.02 & 0.02 \\
EC-Droplet \#C3 & 0.15 & 0.432 & 0 & \\
\hline
\end{tabular}

a

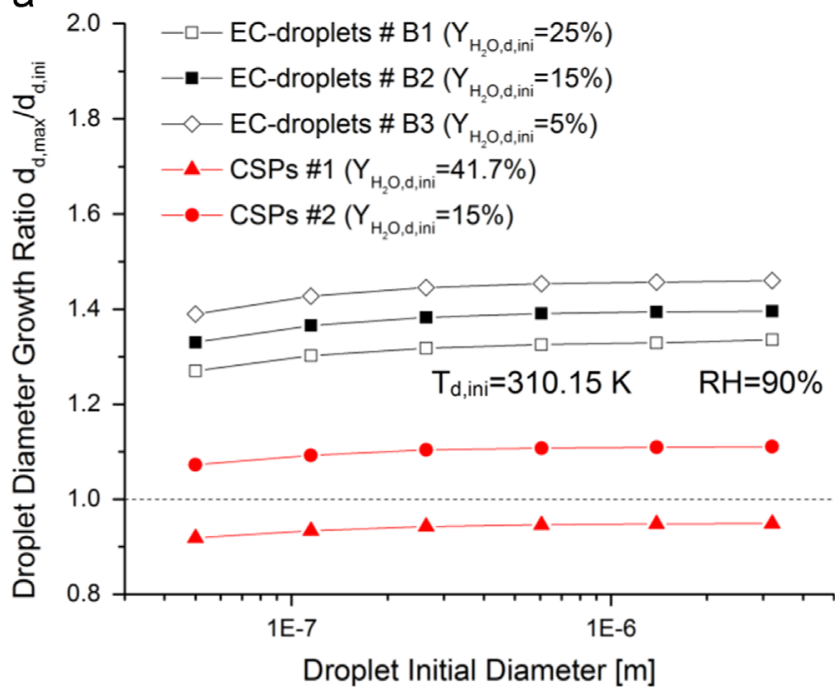

b

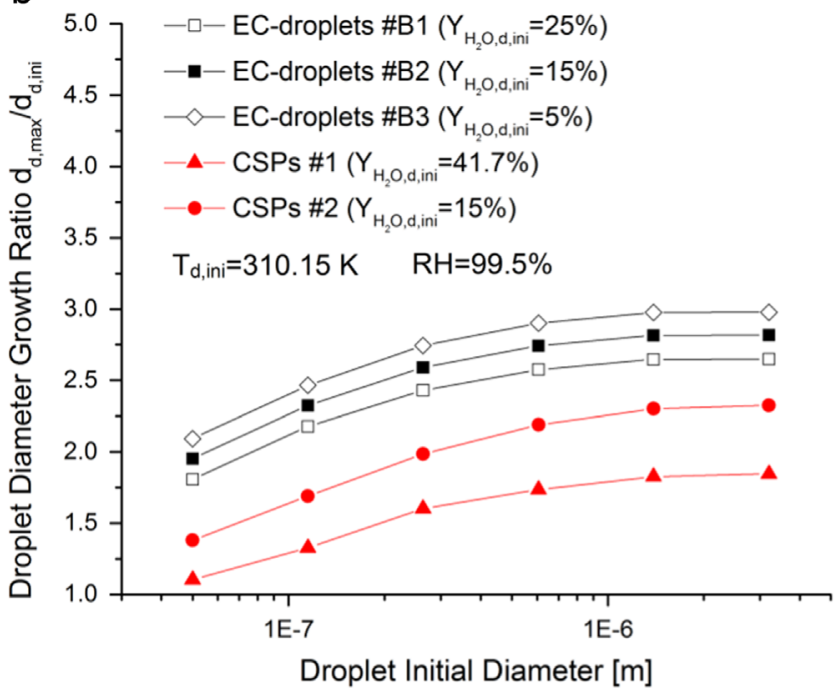

Fig. 4. Droplet diameter growth ratio comparisons between conventional cigarette smoke particles (CSPs \#1 and CSPs \#2) and EC-droplets with different initial water mass fractions: (a) $R H=90 \%$ and (b) $R H=99.5 \%$. 
Specifically, the composition of CSPs \#1 can be found in Section 4, while CSPs \#2 consists of 15\% water, 45\% water soluble compounds with molecular weight of $252 \mathrm{~g} / \mathrm{mol}$, and $40 \%$ insoluble compounds with molecular weight of $267 \mathrm{~g} / \mathrm{mol}$. Parameters such as $R H, T_{d, i n i}, d_{d, i n i}$, and $Y_{s, d, i n i}$, which significantly influence the evaporation/condensation behavior of ECdroplets, have been investigated. For example, the evaporation/condensation characteristics of CSPs and EC-droplets (i.e., \#B1- \#B3) at $T_{\text {env }}=310.15 \mathrm{~K}$ are shown in Fig. 4(a) and (b). At $R H=90 \%$ (see Fig. 4(a)) water vapor condenses at the surfaces of the EC-droplets \#B1-\#B3 with different initial water mass fractions $\left(0.05 \leq Y_{\mathrm{H}_{2} \mathrm{O}, \mathrm{d} \text {,ini }} \leq 0.25\right)$, resulting in different levels of hygroscopic growth. For the specific compositions of the EC droplets (see Table 1), a higher initial water mass fraction

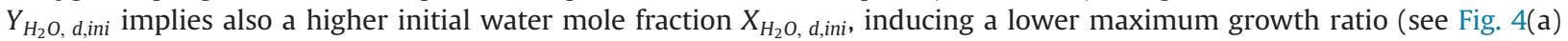
and (b)). Specifically, according to Eq. (9), a higher $X_{\mathrm{H}_{2} \mathrm{O}, d}$ indicates an elevated partial pressure of the water vapor $P_{\mathrm{H}_{2} \mathrm{O}, v s u r f}$ at the droplet surface, thereby lowering the condensation mass flow rate and hence leading to a lower growth ratio. In contrast, water in CSPs \#1 evaporates which leads to droplet-size reduction for all CSPs \#1 with initially different diameters. Specifically, due to the relatively high water mole fraction in the CSPs \#1, i.e., $X_{\mathrm{H}_{2} \mathrm{O}, \text { dini }}>0.9$ (which is because of the high molecular weights of other components in CSPs \#1, i.e., $340 \mathrm{~g} / \mathrm{mol}$ ), the initial partial pressure of water vapor at the droplet surfaces are higher than the ambient partial pressure of water vapor, which is the driving mechanism for evaporation. For CSPs \#2, water condensate on their surfaces and the growth ratios of different initial diameters are lower than all EC-droplets but higher than CSPs \#1. Particularly with the same mass fraction of water (i.e., 15\%), CSPs \#2 grow less than EC-droplets \#B2 (see Fig. 4(a) and (b)). Similarly, EC-droplets and CSPs \#2 growth differences are due to the differences in initial mole fraction of water, while the initial water content and $R H$ were the same for both aerosols. Specifically,
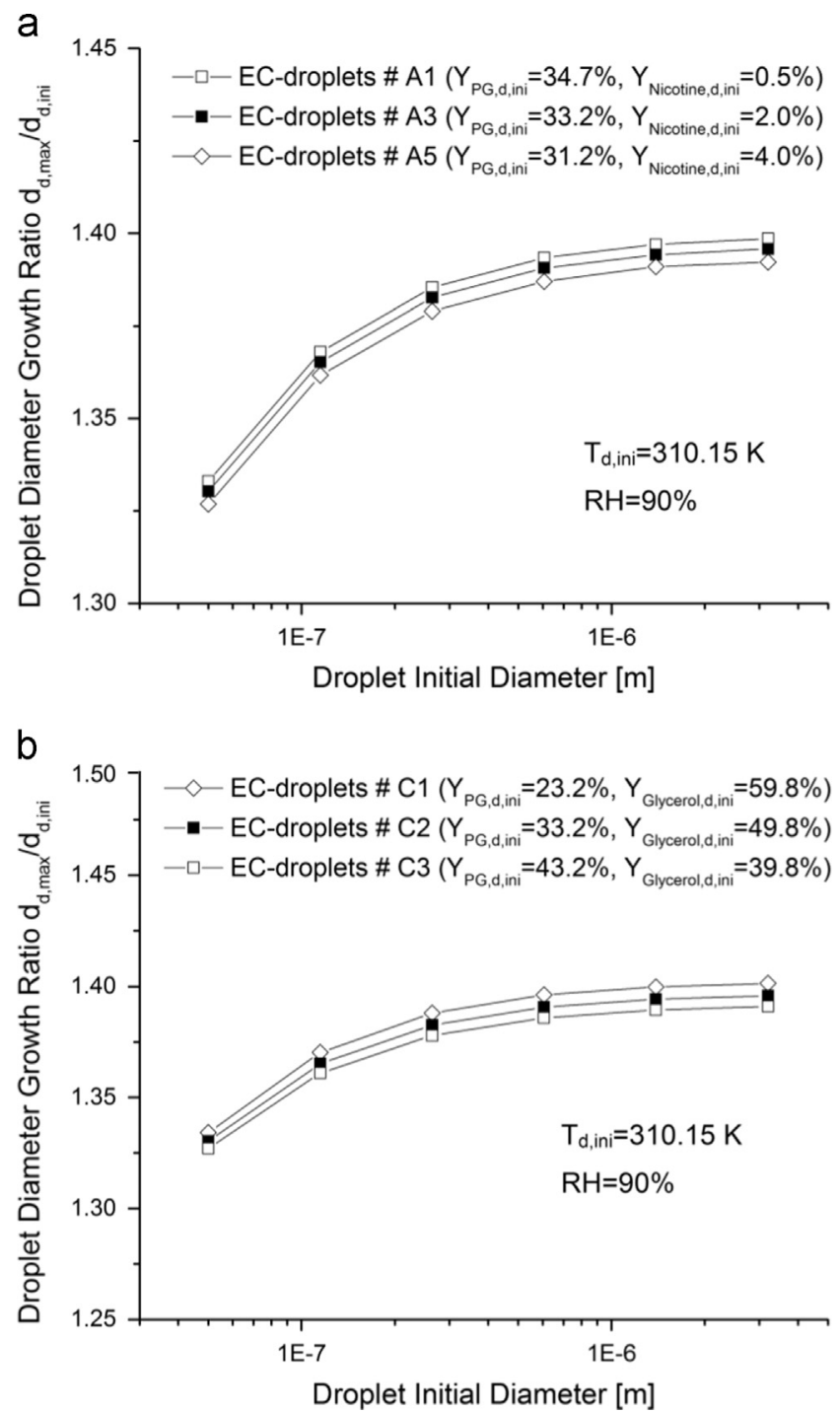

Fig. 5. Droplet diameter growth ratio comparisons among EC-droplets with different initial compositions: (a) different PG and nicotine mass fractions and (b) different PG and glycerol mass fractions. 
calculation of the mole fractions of water in the initial droplet compositions revealed that the initial mole fraction in CSPs \#2 is approximately $70 \%$ and the initial mole fraction of water in the EC-droplets \#B2 is approximately $45 \%$. The different mole fractions are due to the different molecular weights of other components in EC-droplets \#B2 vs. CSPs \#2. Thus, the higher growth ratio of the EC-droplets \#B2 is due to the lower initial mole fraction of water (45\% vs. $70 \%$ ), so that the EC-droplet \#B2 "collects" less water from the ambient than CSPs before they reach quasi-equilibrium (see Eq. (9)). At $R H=99.5 \%$, both EC-droplets and CSPs grow due to the ambient water-vapor condensation effect (Fig. 4(b)). However, CSPs \#1 and \#2 grow less than EC-droplets \#B1- \#B3. It can be concluded that in the same wet-air environment (i.e., water vapor only) and with the same initial droplet diameter, EC-droplets \#B1-B3 will be always larger in size than the liquid CSPs \#1 and \#2. Generalizing this observation, EC-droplets with the same $d_{d, i n i}$ are growing larger than CSPs, and hence with their higher inertia are less affected by the local airflow structure than CSPs.

Clearly, initial droplet diameter and ambient $R H$ values are two dominant parameters influencing the hygroscopic behavior of EC-droplets and CSPs. Specifically, as the $R H$ increases, the maximum droplet diameter growth ratio increases significantly (see Fig. 4(a) vs. Fig. 4(b)). Additionally, at lower initial droplet diameters the maximum droplet diameter growth ratio decreases, because of the Kelvin effect (Hinds, 1999; Fujikawa et al., 2013). The underlying assumption is that the partial pressure of water vapor at the droplet surface, $P_{\mathrm{H}_{2} \mathrm{O} \text {,vsurf }}$, is the saturated pressure of the water in the

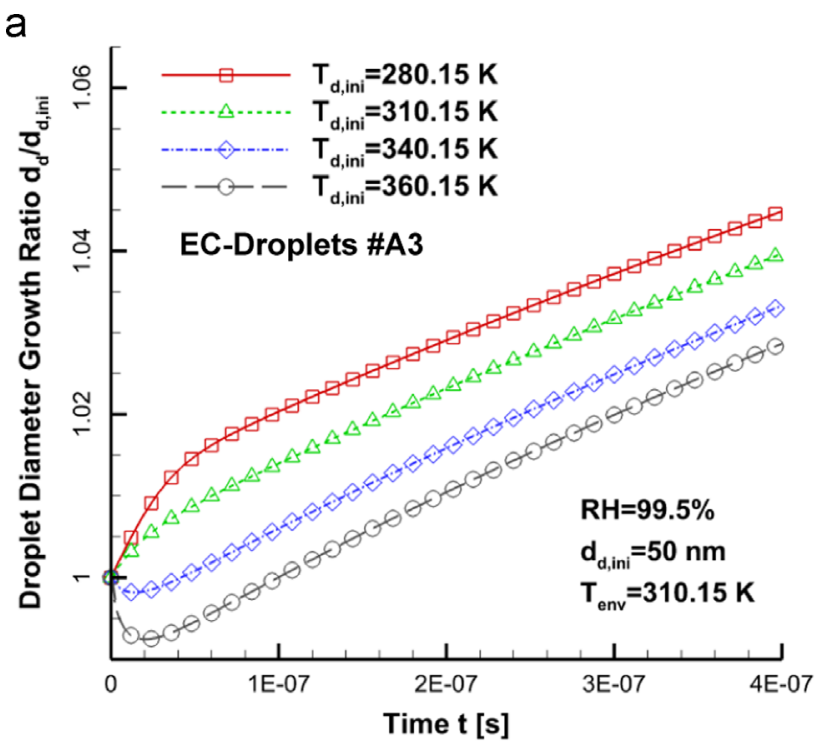

b

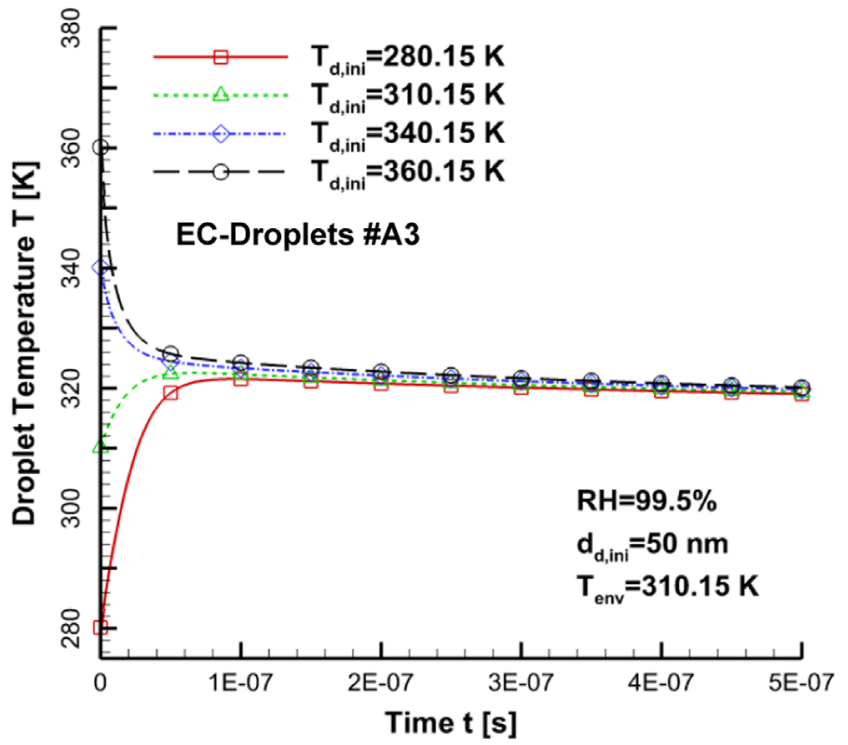

Fig. 6. Initial droplet temperature influence on droplet evaporation/condensation characteristics (EC-droplet \#A3): (a) droplet diameter growth ratio vs. time and (b) droplet temperature vs. time. 
multicomponent droplet. For the droplets with the same composition, $P_{\mathrm{H}_{2} \mathrm{O} \text {,vsurf }}$ is higher in the droplet with smaller size due to the Kelvin effect. As a result, for smaller droplets (e.g., $d_{d, i n i}=50 \mathrm{~nm}$ ) it is easier to reach its maximum size (i.e., quasiequilibrium) than for larger droplets (e.g., $d_{d, i n i}=3200 \mathrm{~nm}$ ).

As given in Table 1, we employed three sets of EC-droplets, i.e., A1-A5, B1-B3, and C1-C3, in order to investigate the influence of initial droplet composition on the evaporation/condensation behavior. Figure 5(a) and (b) shows that the variations in initial PG, glycerol and nicotine concentrations in the droplet have a minor impact on the droplet diameter growth ratio, because of their low volatilities when compared to water.

Figure 6(a) and (b) visualizes for EC-droplet \#A3, subject to $R H=99.5 \%$ and $T_{\text {env }}=310.15 \mathrm{~K}$, the influence of the initial droplet temperature $\left(280.15 \mathrm{~K} \leq T_{\text {dini }} \leq 360.15 \mathrm{~K}\right)$ on both droplet growth and droplet temperature. The initial droplet temperature has only a minor impact on droplet growth and droplet temperature evolution, except during an initial period of $t<0.5 \mathrm{e}-07 \mathrm{~s}$. Based on the fact that the vapor pressure increases as the temperature increases, if the initial droplet temperature is high enough (e.g. $T_{d, i n i}=360.15 \mathrm{~K}$ ), the water in the droplet will initially evaporate due to the larger partial pressure at the droplet surface when compared to the ambient water vapor partial pressure, leading to a reduction in droplet size (see Fig. 6(a)). However, lower initial droplet temperatures (e.g. $T_{d, i n i}=280.15 \mathrm{~K}$ ) result in lower partial pressures at the droplet surfaces, thereby causing higher condensation rates (see Fig. 6(a)). Nevertheless, the time duration of this process is very short and hence could be neglected. Figure 6(b) shows that the droplet temperature rapidly approaches the ambient temperature, regardless of the $T_{d, i n i}$ value. For this reason, it is not expected that the initial droplet temperature is an essential parameter influencing EC-droplet evaporation/condensation behavior.

\subsection{Correlation of EC-droplet growth ratio for realistic conditions}

Focusing on realistic conditions inside the human respiratory system (say, $R H=99.5 \%$ and $T_{\text {env }}=310.15 \mathrm{~K}$ ), a correlation is provided based on our numerical results for EC-droplet growth ratios (see Table 1 for droplet compositions). The correlation appears as a function of $d_{d, \text { ini }}$ and $Y_{H_{2} O}$, d,ini, which may be employed for future comparisons with experimental measurements.
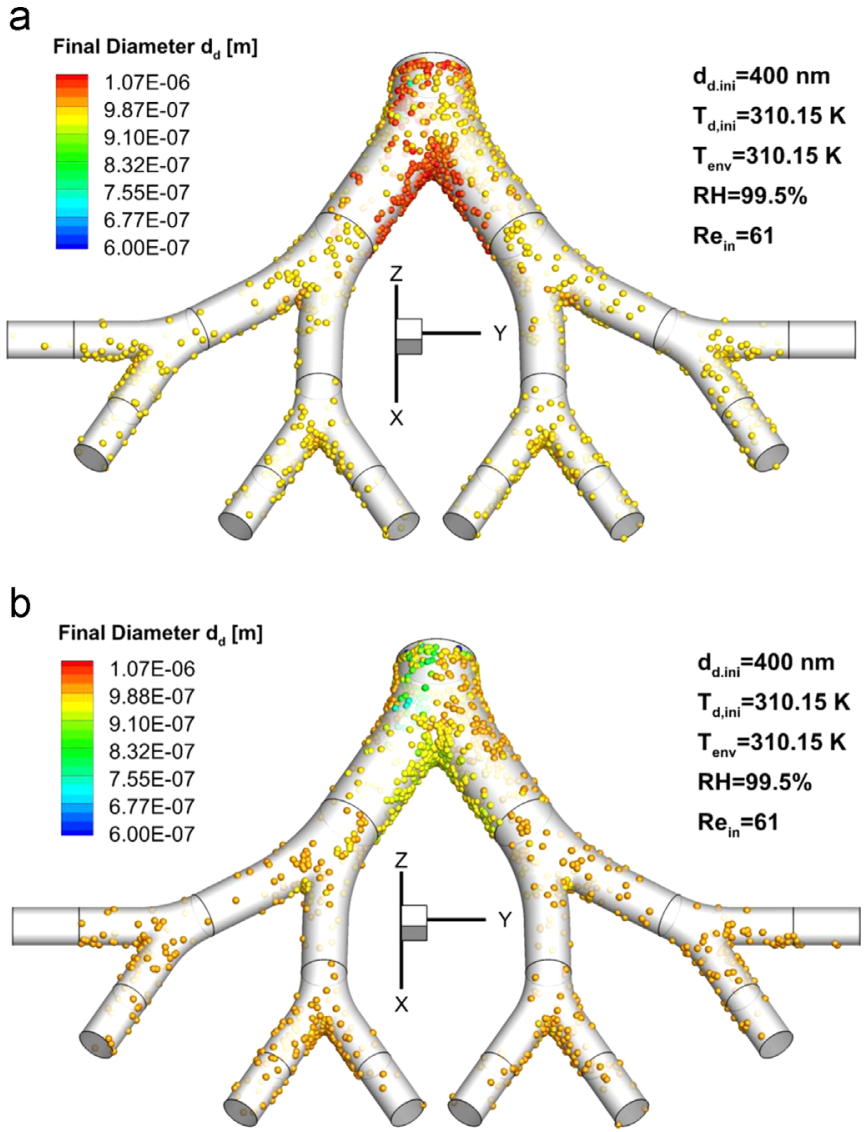

Fig. 7. Effect of droplet temperature modeling on droplet hygroscopic growth and deposition patterns for EC-droplets \#A3 in G3-G6 TBU: (a) variable droplet temperature and (b) isothermal droplet. (For interpretation of the references to color in this figure, the reader is referred to the web version of this article.) 
According to the parametric analyses, only the droplet water concentration influences significantly the hygroscopic characteristics. Thus, neglecting the influence of the other components, we interpolated our numerical results in Fig. 4(b) to generate a hygroscopic growth prediction for representative EC-droplet \#A3 in the form:

$$
\frac{d_{d, \text { max }}}{d_{d, \text { ini }}}=\left(\frac{C_{1}}{d_{d, \text { ini }}}+C_{2}\right)\left(C_{3} Y_{\mathrm{H}_{2} \mathrm{O}, \mathrm{d}, \text { ini }}^{2}+C_{4} Y_{\mathrm{H}_{2} \mathrm{O}, \mathrm{d}, \mathrm{ini}}+C_{5}\right)
$$

Here, $C_{1}=-6.166 e-8, C_{2}=3.876, C_{3}=-0.07979, C_{4}=-0.4166$, and $C_{5}=0.7869$. Equation (13) is valid for $d_{d, i n i} \in$ $[50 \mathrm{~nm}, 3200 \mathrm{~nm}]$ and $Y_{\mathrm{H}_{2} \mathrm{O}, \text { dini }} \in[0.05,0.25]$. The coefficient of determination $r^{2}$ is 0.9776 .

\subsection{Transport, growth and deposition of EC-droplets, liquid CSPS and solid Particles in the G3-G6 TBU}

In this section, the transport and deposition of EC-droplets \#A3, CSPs and solid particles are discussed, assuming the same initial particle diameters (i.e., $264 \mathrm{~nm} \leq d_{d, i n i} \leq 3200 \mathrm{~nm}$ ) in the $\mathrm{G} 3-\mathrm{G} 6 \mathrm{TBU}$. The inlet surface is in plane $x=0$, and the coordinate of inlet centroid is $(0,0,0)$. The airflow mainstream direction is along the positive $x$-axis. By neglecting the gravity effect, all the depositions are caused by inertial impaction as well as secondary flow and Brownian diffusion. The discrete-phase time step $\Delta t_{d}$ was set much smaller than the default value assigned by ANSYS Fluent 14.5 (i.e., $\Delta t_{d}=0.1 e-6$ seconds for droplets with $d_{d, i n i}=400 \mathrm{~nm}$ ) in order to guarantee that droplet-temperature calculations convergence and accurate Brownian motion effects were obtained.

\subsubsection{Influence of droplet temperature modeling on hygroscopic behavior}

When trying to reduce computer-run time, one consideration is to assume a constant droplet temperature and hence ignoring Eq. (10). As an example, Fig. 7(a) and (b) contrasts the local deposition patterns of EC-droplet \#A3 $\left(d_{d, i n i}=400 \mathrm{~nm}\right)$ with and without solving the droplet energy conservation equation (see Eq. (11)), where the deposited droplets are colored by their final diameters. The images indicate that although the local droplet deposition patterns in G3-G6 are somewhat similar for both cases, assuming a constant droplet temperature (see Fig. 7(b)) generates slightly larger maximum droplet diameters. Specifically, when considering a realistic (initial) droplet temperature change, the water vapor condensation rate is lower compared to the case of constant droplet temperature. Therefore, solving the droplet energy conservation equation is necessary for an accurate prediction of droplet hygroscopic characteristics and hence droplet transport and deposition.

\subsubsection{Deposition efficiency comparisons for EC-droplets, CSPs, and solid particles}

5.3.2.1. Total deposition efficiency. Figure 8 compares simulation results in terms of total deposition efficiency (TDE) for EC-droplet \#A3, liquid CSPs \#1, and solid particles $\left(264 \mathrm{~nm} \leq d_{d, i n i} \leq 3200 \mathrm{~nm}\right.$ ). In order to show the hygroscopic growth influence on droplet deposition, we selected CSPS \#1 instead of CSPs \#2 because of the larger growth ratio differences between CSPs \#1 and EC-droplet \#A3. It can be observed that with the same initial particulate diameter, EC-droplets \#A3 deposit less than CSPs \#1 and solid particles, while CSPs \#1 deposit less than solid particles. This is because the hygroscopic growth of droplets during their transport limits Brownian diffusion, thereby reducing TDE in G3-G6. Also, because the growth ratio of CSPs \#1 is less than \#A3 EC-droplets (see Fig. 4(b)), the hygroscopic limiting effect on Brownian diffusion of CSPs is less than for EC-droplets, thereby providing higher TDE-values when compared to EC-droplets. It can be concluded that CSPs \#1 deposit more in the G3-G6 TBU than \#A3 EC-droplets, due to the stronger Brownian diffusion effect induced by

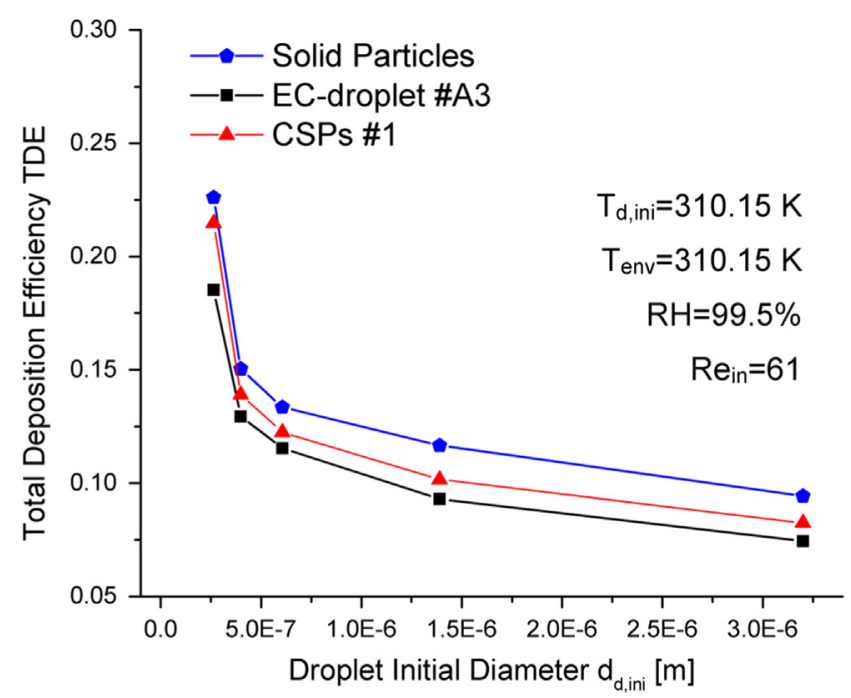

Fig. 8. Total deposition efficiencies (TDEs) of EC-droplet \#A3, CSPs \#1 and solid particles with different initial diameters $\left(264 \mathrm{~nm} \leq d_{d, i n i} \leq 3200 \mathrm{~nm}\right)$ in the G3-G6 TBU. 

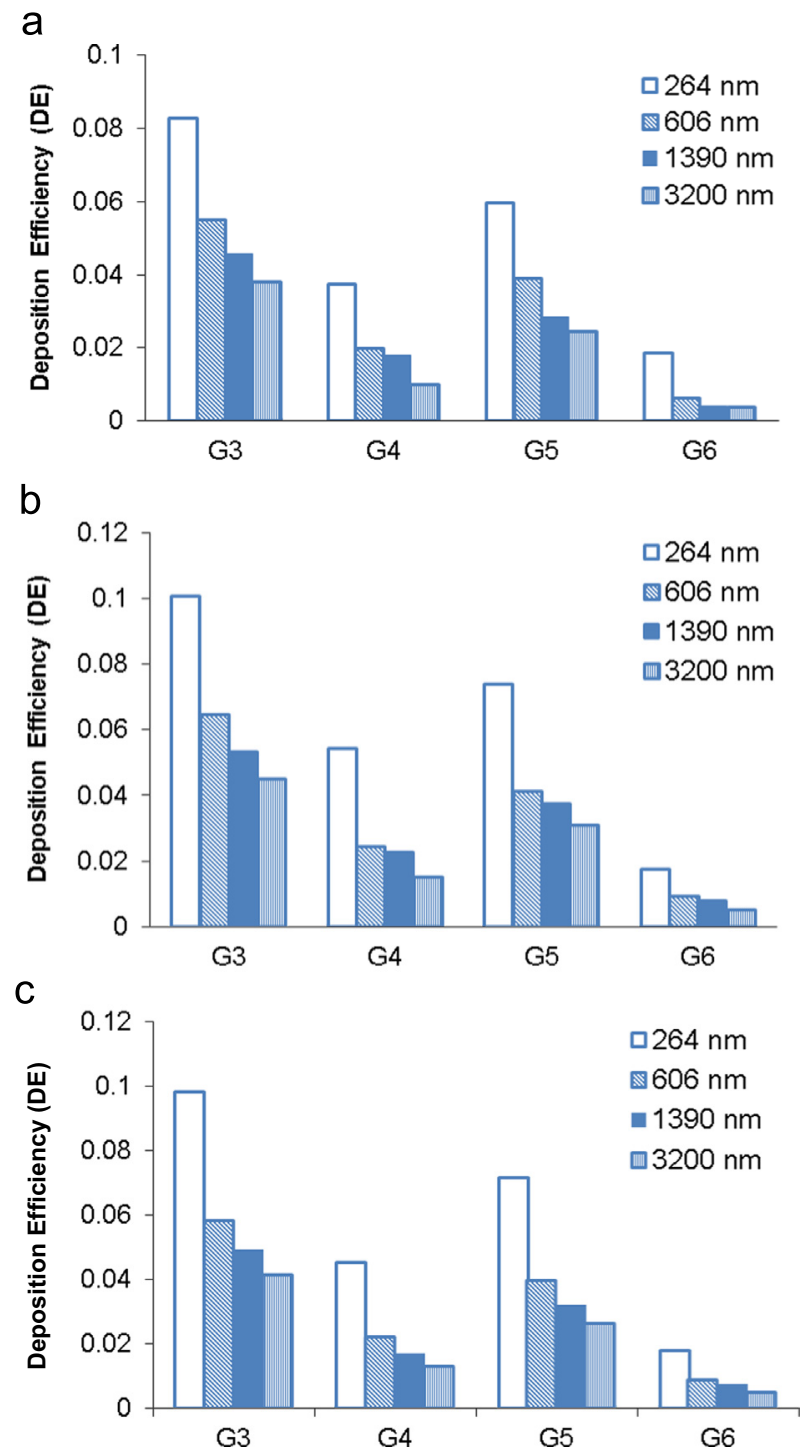

Fig. 9. Regional deposition efficiencies (RDEs) of EC-droplet \#A3 and solid particles with different initial diameters (264 $\left.\mathrm{nm} \leq d_{d, i n i} \leq 3200 \mathrm{~nm}\right)$ in $\mathrm{G} 3-\mathrm{G} 6$ ( $T_{d, i n i}=$ $310.15 \mathrm{~K}, \quad T_{\text {env }}=310.15 \mathrm{~K}, \quad R H=99.5 \%, \quad R e_{\text {in }}=61$ ): (a) EC-droplets \#A3; (b) solid particles; and (c) CSPs \#1.

lower hygroscopic growth. It can be conjectured that EC-droplets may deposit up to the first bifurcation more than CSPs \#1 due to inertial impaction (Feng \& Kleinstreuer, 2013). However, EC-droplets tend to deposit more in G3-G6 TBUs and other human lung airway regions where Brownian diffusion is a more dominating depositing mechanism for nano-size droplets.

5.3.2.2. Regional deposition efficiency. A regional deposition efficiency (RDE) evaluation within G3-G6 was also performed. Figure 9(a)-(c) illustrates RDEs for EC-droplets \#A3, CSPs \#1, and solid particles in G3-G6 with a variety of initial droplet/ particle diameters $\left(264 \mathrm{~nm} \leq d_{d, i n i} \leq 3200 \mathrm{~nm}\right.$ ). Consistent with Fig. 8, data in Fig. 9(a)-(c) indicate that hygroscopic droplet growth also reduces the RDEs in all TBU-regions when compared to solid particles, due to weaker Brownian diffusion of the droplets as they are increasing in size. Specifically, with no gravity and relatively low inlet Reynolds number $\left(R e_{i n}=61\right)$, Brownian motion is the major deposition mechanism, as especially manifested with the smaller nano-droplets. Although the hygroscopic behavior of the droplets can increase their inertia and hence impaction, it reduces the RDE by lowering the Brownian motion intensities of the discrete phase. Through the simulation results shown in Fig. 9(a)-(c) we also found that for higher initial droplet diameters, $d_{d, i n i}$, EC-droplets \#A3, CSPs \#1 and solid particles all deposit less regionally, again because of reduced Brownian motion for the larger particulate matter.

5.3.3. Transport, growth, and deposition characteristics of EC-droplets

5.3.3.1. Local deposition patterns. Different Lagrangian-time dependent aspects of EC-droplets \#A3 (i.e., droplet final diameter; droplet travel time, and droplet initial distance from the inlet centroid) are displayed in Figs. 10(a)-(c) and 11(a)-(c) for initial 
a
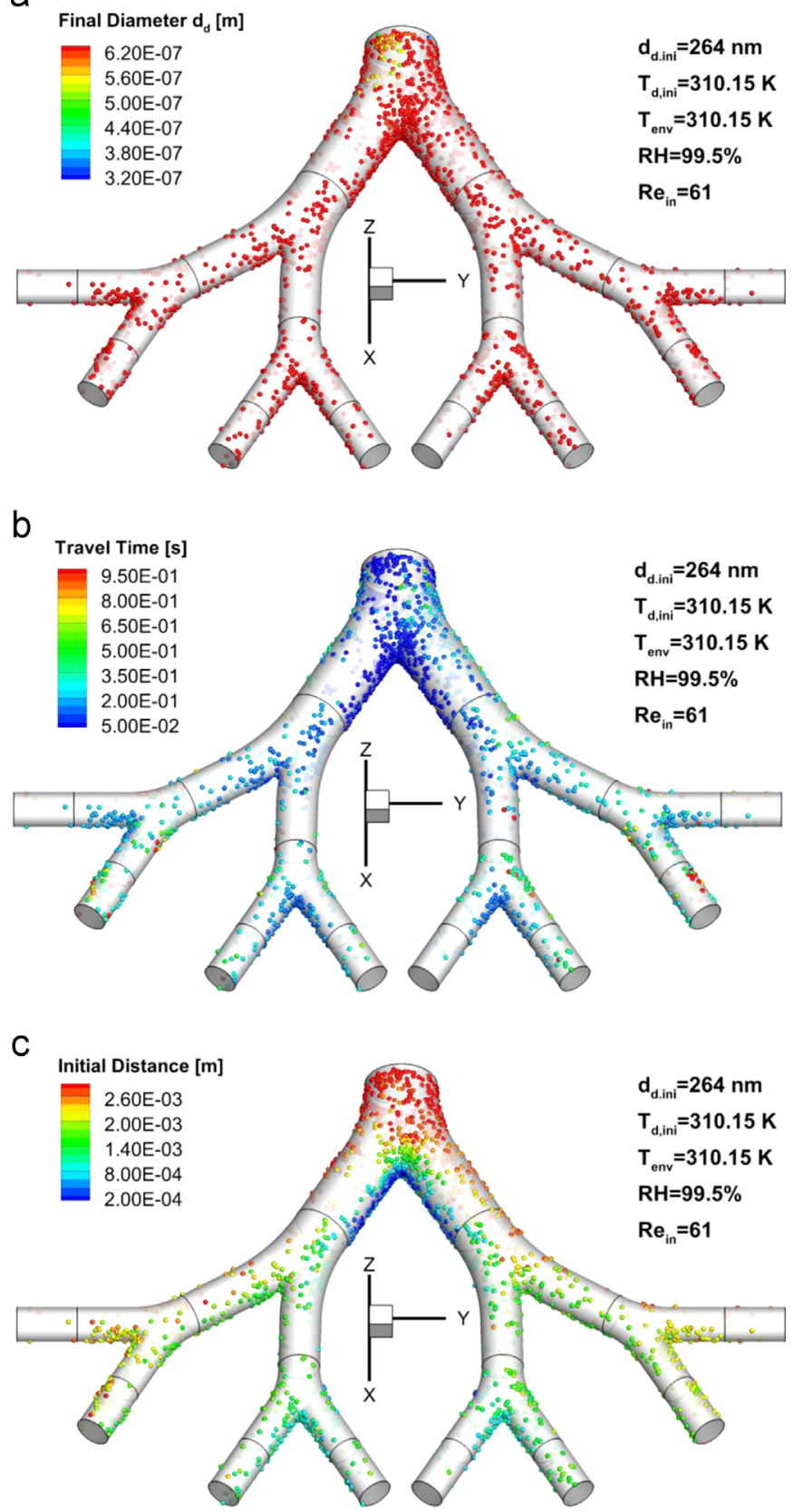

Fig. 10. Local deposition patterns of EC-droplet \#A3 $\left(d_{d, \text { ini }}=264 \mathrm{~nm}\right.$ ) colored by different variables: (a) final droplet diameter; (b) droplet travel time before deposition; and (c) initial distance between the droplet and the inlet centroid. (For interpretation of the references to color in this figure legend, the reader is referred to the web version of this article.)

diameters $264 \mathrm{~nm}$ (Fig. 10) and $3200 \mathrm{~nm}$ (Fig. 11). In case of the smaller droplets, due to strong diffusion effects, more dispersed deposition patterns can be observed. Also, for larger droplets inertial impaction becomes more significant, which results in the more concentrated deposition patterns in G5 when compared to G3.

5.3.3.2. Droplet initial diameter effect. According to Figs. 10(a) and 11(a), we found that EC-droplets with smaller $d_{d, \text { ini }}$ approach the quasi-equilibrium hygroscopic status faster than those with larger $d_{d, \text { ini }}$ values. Specifically, in G3-G6, $264 \mathrm{~nm}$ EC-droplets reach the hygroscopic equilibrium before traveling to the first bifurcating point (see Fig. 10(a)) while $3200 \mathrm{~nm}$ EC-droplets keep growing until the G6 outlets (see Fig. 11(a)), which is caused by the Kelvin effect. As mentioned, more dispersed deposition patterns can be observed for smaller droplets compared to droplets of larger size. Again, Brownian diffusion contributes more significantly to smaller EC-droplet deposition when compared to direct impaction. 


\section{a}

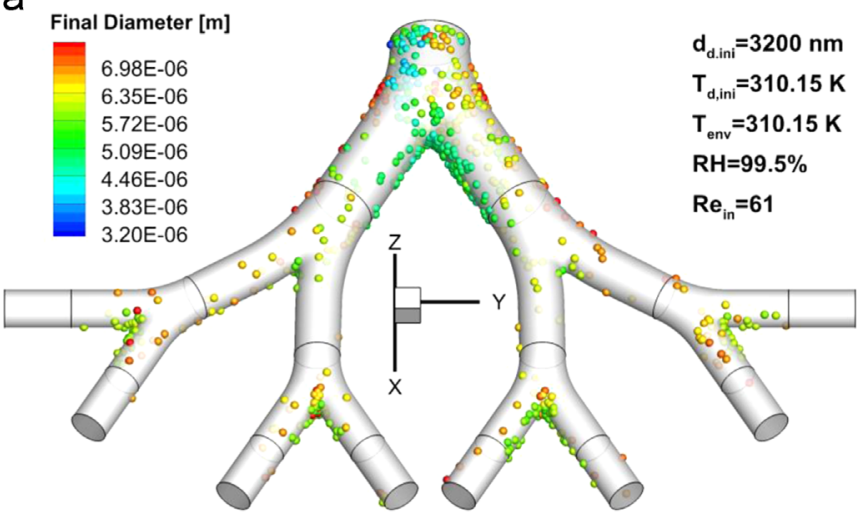

b
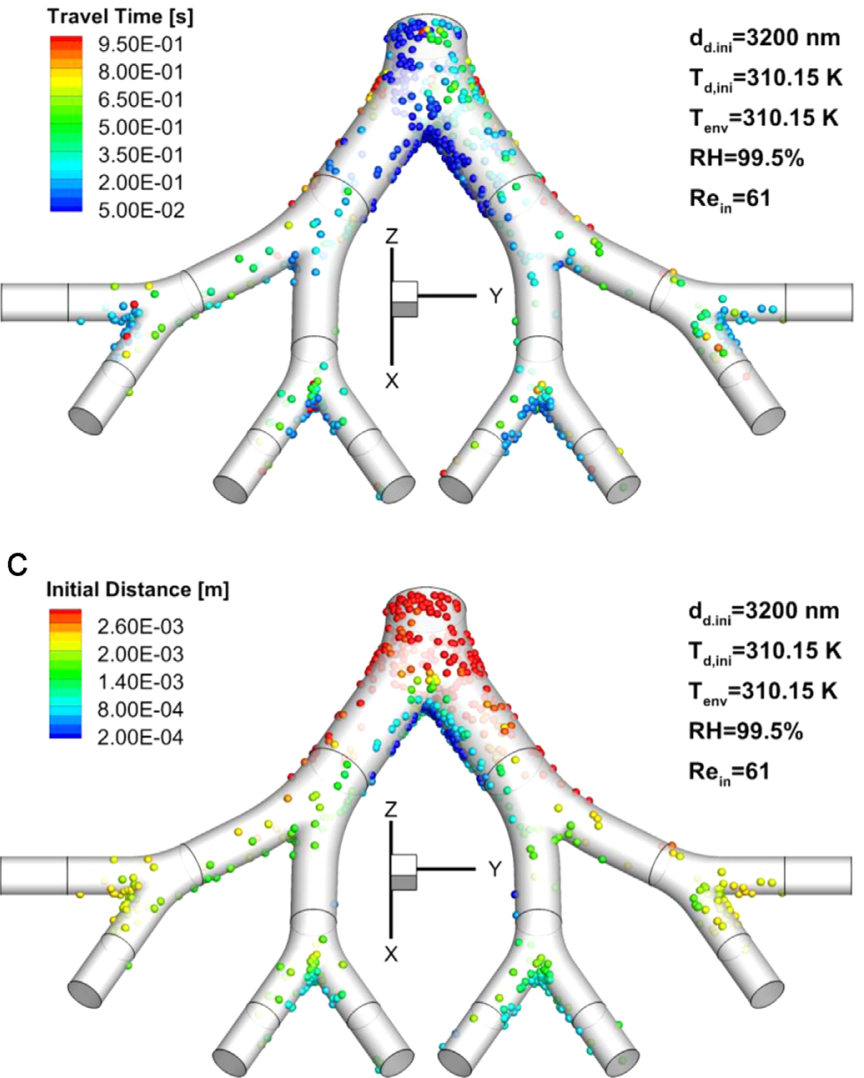

Fig. 11. Local deposition patterns of EC-droplet \#A3 $\left(d_{d, i n i}=3200 \mathrm{~nm}\right)$ colored by different variables: (a) final droplet diameter; (b) droplet travel time before deposition; and (c) initial distance between the droplet and the inlet centroid. (For interpretation of the references to color in this figure legend, the reader is referred to the web version of this article.)

5.3.3.3. Droplet deposition mechanisms vs. droplet travel time. Droplet travel time can be considered as a variable to differentiate deposited droplets with different depositing mechanisms. Figures 10(b) and 11(b) show the deposition patterns of EC-droplets colored by their travel time before they deposit. We found that due to Brownian motion many droplets, being initially positioned close to the wall, deposit within a short time in the location near the inlet because of the strong diffusion (see the dark blue droplets near the inlet in Figs. 10(b) and 11(b)). Also, the travel time of most droplets depositing near the G3 bifurcating point is relatively short, which is due to their elevated velocity obtained from the core of the Poiseuille velocity profile. This conclusion is also supported by Figs. 10(b) and 11(b), which shows that the droplets depositing near the G3 bifurcating point are initially located near the inlet centroid. After transport to the locations close to the G3 bifurcating point, diffusion and direct impaction both serve as the mechanisms for deposition. Furthermore, it can be observed that some droplets deposit in all regions with relatively longer travel times than others. Their deposition is caused by diffusion, 
i.e., droplets initially located near the wall will transport slowly downstream, indicating longer travel time and negligible inertial impaction (see the red droplets in Figs. 10(b) and 11(b) as examples).

5.3.3.4. Deposition location vs. initial droplet position. Figures 10 (c) and 11 (c) display the droplet deposition patterns colored by their initial distance to the G3 inlet centroid (i.e., $0 \leq h_{\mathrm{G} 3} \leq 0.003 \mathrm{~m}$ ). We observed most droplets with $h_{\mathrm{G} 3}>0.0026 \mathrm{~m}$ deposit in G3 before the bifurcating point due to the Brownian motion effect, while most droplets with $h_{\mathrm{G} 3}<0.001 \mathrm{~m}$ deposit at the G3 inner walls downstream of the G3 bifurcating point. For droplets with $0.001 \mathrm{~m}<h_{\mathrm{G} 3}<0.0026 \mathrm{~m}$, their deposition locations appear throughout the TBU. Depending on their initial position, droplets within $0.001 \mathrm{~m}<h_{\mathrm{G} 3}$ $<0.0026 \mathrm{~m}$, i.e., with a closer position to the inlet centroid, will transport and deposit in locations closer to the centerline of the TBU.

\section{Summary}

A computer simulation model based on the novel Multicomponent Mixture plus Discrete Droplet (MM-DD) modeling approach has been proposed. It allows for the prediction of the transport and deposition of inhaled EC-droplets and liquid CSPs in light of simultaneous multicomponent droplet-vapor interactions. We numerically studied the droplet size-change dynamics and discovered that due to the combined multicomponent evaporation/condensation effects, all EC-droplets will undergo size-changes due to following processes:

(1) Vaporization/condensation of a droplet will be influenced by its initial temperature for a negligible time duration after the droplet has been released from the inlet.

(2) After the droplet temperature $T_{d, \text { ini }}$ quickly approaches the ambient temperature $T_{\text {env }}$, water vapor start to condensate at the droplet surface, leading to hygroscopic growth, i.e., droplet-size increase. Meanwhile, the other components (i.e., glycerol, PG, and nicotine) keep evaporating slowly due to the absence of their vapor species surrounding the droplet and their low volatilities.

(3) Droplets reach their maximum size at a certain time when the water in the droplet is saturated.

(4) Afterwards, the vaporization of other components and the adjustment of water in the droplet will continuously decrease the droplet size.

(5) For conventional smoke particles (liquid CSPs), we found that they will grow to maximum sizes less than EC-droplets (i.e., types \#B1-\#B3) with the same initial diameters, indicating stronger Brownian motion effects during their transport.

(6) Parameters $R H, d_{d, \text { ini }}$ and $Y_{\mathrm{H}_{2} \mathrm{O}, \text { ini }}$ can significantly influence the EC droplet-growth ratio. It indicates that due to the low volatilities of PG, glycerol and nicotine, their compositions can be modified (within certain value-ranges) with no significant influence on the EC-droplet's hygroscopic behavior.

(7) For the typical values of $\mathrm{RH}=99.5 \%$ and $T_{e n v}=310.15 \mathrm{~K}$, a new correlation based on our numerical results for ECdroplets' hygroscopic growth prediction is given. The diameter-ratio correlation appears as a function of $d_{d, \text { ini }}$ and

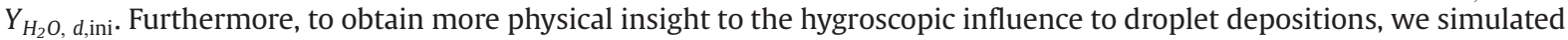
EC-droplet \#A3 dynamics with different initial diameters $\left(264 \mathrm{~nm} \leq d_{d, i n i} \leq 3200 \mathrm{~nm}\right)$. We compared the deposition patterns of such droplets with those of the CSPs and solid particles in order to evaluate the hygroscopic influences. Our data indicate the following:

(a) Due to Brownian diffusion, smaller droplets deposit more in the G3-G6 TBU.

(b) Brownian motion of submicron droplets induce dispersed deposition patterns, while inertial impaction results in concentrated deposition patterns, i.e., near bifurcating points.

(c) Hygroscopic growth of droplets will influence their deposition patterns in three ways by

- enhancing the inertial effects,

- limiting the Brownian motions,

- influencing regional depositions in complex ways.

For future work, the overall goal is to use the MM-DD model presented in Section 2 for the prediction of interacting airdroplet-vapor dynamics from inhaled EC-aerosols in representative whole lung models.

\section{Acknowledgments}

Research described in this article was supported in part by Altria Client Services Inc. (Richmond, VA). The authors thank Dr. Yezdi B. Pithawalla for helpful discussions and for reviewing the manuscript. The authors also acknowledge the financial support of Yu Feng by a grant from NSF (CBET-1232988), Environmental Health and Safety of Nanotechnology, Dr. Barbara Karn, Program Director. The use of both Fluent and CFX software from ANSYS Inc. (Canonsburg, PA, USA) is gratefully acknowledged as well. 


\section{References}

Berthier, J., \& Silberzan, P. (2006). Microfluidics for Biotechnology. Artech House: MA, USA.

Brown, C.J., \& Cheng, J.M. (2014). Electronic cigarettes: product characterization and design considerations. Tobacco Control, 23(Suppl. 2), ii4-ii10.

Cheng, T. (2014). Chemical evaluation of electronic cigarettes. Tobacco Control, 23(Suppl. 2), ii11-ii17.

Chen, I.L. (2013). FDA summary of adverse events on electronic cigarettes. Nicotine E' Tobacco Research, 15(2), 615-616.

Chen, I.L., \& Husten, C.G. (2014). Introduction to tobacco control supplement. Tobacco Control, 23, ii1-ii3.

Feng, Y., \& Kleinstreuer, C. (2013). Analysis of non-spherical particle transport in complex internal shear flows. Physics of Fluids, $25(9), 091904$.

Feng, Y., \& Kleinstreuer, C. (2014). Micron-particle transport, interactions and deposition in triple lung-airway bifurcations using a novel modeling approach. Journal of Aerosol Science., 75, 1-15.

Fujikawa, S., Yano, T., \& Watanabe, M. (2013). Vapor-Liquid Interfaces, Bubbles and Droplets. Springer-Verlag: Berlin, Heidelberg, Germany.

Hinds, W.C. (1999). Aerosol Technology: Properties, Behavior, and Measurement of Airborne Particles 2nd ed.). John Wiley \& Sons: New York, USA.

Ishizu, Y., Ohta, K., \& Okada, T. (1980). The effect of moisture on the growth of cigarette-smoke particles. Beitrage zur Tabakforschung International, 10(3), $161-168$.

Li, W., \& Hopke, P.K. (1993). Initial size distributions and hygroscopicity of indoor combustion aerosol particles. Aerosol Science and Technology, 19(3), 305-316.

Longest, P.W., \& Xi, J. (2008). Condensational growth may contribute to the enhanced deposition of cigarette smoke particles in the upper respiratory tract. Aerosol Science and Technology, 42(8), 579-602.

Polosa, R., Rodu, B., Caponnetto, P., Maglia, M., \& Raciti, C. (2013). A fresh look at tobacco harm reduction: the case for the electronic cigarette. Harm Reduction Journal, 10(1), 19.

Sirignano, W.A. (2010). Fluid Dynamics and Transport of Droplets and Sprays2nd ed.). Cambridge University Press: New York, NY, USA.

Tu, H.H., \& Ray, A.K. (2005). Measurement of activity coefficients from unsteady state evaporation and growth of microdroplets. Chemical Engineering Communications, 192, 474-498.

Turns, S.R. (1996). An Introduction to Combustion, vol. 287. McGraw-Hill: New York, NY, USA.

Weibel, E.R. (1963). Morphometry of the Human Lung. Academic Press: New York, NY, USA.

Zhang, Z., \& Kleinstreuer, C. (2001). Effect of particle inlet distribution on deposition in a triple bifurcation lung airway model. Journal of Aerosol Medicine, 14 (1), 13-29.

Zhang, Z., Kim, C.S., \& Kleinstreuer, C. (2006a). Water vapor transport and its effects on the deposition of hygroscopic droplets in a human upper airway model. Aerosol Science and Technology, 40(1), 1-16.

Zhang, Z., Kleinstreuer, C., \& Kim, C.S. (2006b). Isotonic and hypertonic saline droplet deposition in a human upper airway model. Journal of Aerosol Medicine, 19(2), 184-198.

Zhang, Z., Kleinstreuer, C., \& Hyun, S. (2012a). Size-change and deposition of conventional and composite cigarette smoke particles during inhalation in a subject-specific airway model. Journal of Aerosol Science, 46, 34-52.

Zhang, Z., Kleinstreuer, C., \& Feng, Y. (2012b). Vapor deposition during cigarette smoke inhalation in a subject-specific human airway model. Journal of Aerosol Science, 53, 40-60. 